\title{
Diagnostic Implication of Mean Platelet Volume in Thrombocytopenia
}

\author{
Archana Bhat*, Christina Goveas and Jayaprakash C S
}

Dept of Pathology, Father Muller Medical College, Mangalore, Karnataka, India

\begin{abstract}
Introduction: The newer haematology analysers measure the platelet indices such as the mean platelet volume (MPV), platelet large cell ratio and platelet distribution width. It has been observed that MPV is increased in conditions associated with thrombocytopenia due to peripheral destruction.

This study was carried out to know the utility of MPV and derive cut-off values in categorizing the conditions causing thrombocytopenia into hyperdestructive and hypoproliferative groups.

Methods: The thrombocytopenia cases were categorized into two groups as hyperdestructive and hypoproliferative groups based on the bone marrow findings. The MPV on the day of admission was taken into consideration. The data were analysed using the mean, standard deviation and the t-test. ROC curve was plotted to derive the cut-off MPV value.

Results: Of the total 89 thrombocytopenia cases, 53 were of hyperdestructive etiology and 36 were of hypoproliferative etiology. The mean MPV for group A was 9.56 with a standard deviation of 1.34 and that for group B was 8.45 with a standard deviation of 1.30 . The $\mathrm{p}$ value was $<0.001$. From the ROC curve, a cut off of $8.5 \mathrm{fl}$ was taken.

Conclusion: We conclude that a MPV value of $>8.5 \mathrm{fl}$ is suggestive of a hyperdestructive etiology. Even though bone marrow remains a gold standard to distinguish between the hyperdestructive and hypoproductive thrombocytopenia groups, platelet indices like MPV can be used to aid in the diagnosis of etiology of thrombocytopenia.
\end{abstract}

Keywords: Mean Platelet Volume, Thrombocytopenia, Platelet Count

\section{Introduction}

Platelet count below the normal levels defines thrombocytopenia. Thrombocytopenia can be caused due to decreased production of platelets or due to increased destruction. The mechanism leading to thrombocytopenia can be ascertained by bone marrow examination which shows megakaryocytic hyperplasia in cases with increased peripheral destruction of platelets and megakaryocytic hypoplasia in cases with decreased production.

The newer haematology analysers measure the platelet indices such as the mean platelet volume (MPV), platelet large cell ratio and platelet distribution width along with the routine blood parameters. On a peripheral smear examination, the platelets measure 1 to 4 microns in diameter. ${ }^{[1]}$ The normal MPV ranges from 7 to $11.7 \mathrm{fl} .{ }^{[2,3]}$

It has been observed that MPV is increased in conditions associated with thrombocytopenia due to destruction of platelets. ${ }^{[4]}$ The bone marrow compensates for the peripheral destruction by release of immarure platelets into the peripheral blood similar to the way reticulocytes are released when there is hemolysis. These immature platelets are larger in size and result in increased MPV.
This study was carried out to know the utility of MPV in categorizing the conditions causing thrombocytopenia into hyperdestructive and hypoproliferative groups.

\section{Materials and Methods}

This was an observational descriptive study conducted in the Department of Pathology of a tertiary care hospital. The institution ethics committee clearance was obtained. The study included 89 cases of thrombocytopenia who have undergone bone marrow evaluation. The data and slides of these cases were retrieved from the archives. The cases were categorized into two groups based on the bone marrow findings. Group A i.e. the hyperdestructive group, included the cases of thrombocytopenia due to excess destruction of platelets whose bone marrow showed normal or increased megakaryopoiesis. Group B i.e. the hypoproliferative group, included the cases of thrombocytopenia due to reduced production of platelets whose bone marrow showed decreased megakaryopoiesis. The MPV on the day of admission was taken into consideration for comparison between the two groups. The data were entered in excel sheet and analysed using the mean, standard deviation and the t-test. 


\section{Results}

The subjects in our study were of the age ranging upto 80 years with maximum of them falling in the age group of 40 to 60 years. Of the total 89 cases, 44 were females and 45 were males.

In our study, of the thrombocytopenia cases due to hyperdestructive etiology $(n=53)$, majority of them showed hyperplastic marrow with erythroid hyperplasia and megakaryocytic hyperplasia and six cases of idiopathic thrombocytopenic purpura(ITP) were noted.

Of the thrombocytopenia cases due to hypoproductive etiologies $(n=36)$, the cases were mostly leukemias
(AML, ALL, APMML), one case was of metastatic adenocarcinoma and 4 cases of plasma cell myeloma.

On statistical analysis, the mean MPV for group A was 9.56 with a standard deviation of 1.34 . The mean MPV for group B was 8.45 with a standard deviation of 1.30 . the $\mathrm{p}$ value was $<0.001$ which was highly significant. The Receiver operating characteristic (ROC) curve was plotted with the co-ordinates (in Table 1) as shown below.

From the ROC curve, a cut off of $8.5 \mathrm{fl}$ was taken for distinguishing between

hyperdestructive and hypoproliferative etiologies, at a sensitivity of $80 \%$ and specificity of $66 \%$.

Table 1: Coordinates of the receiver operating characteristic curve.

\begin{tabular}{|c|c|c|}
\hline Positive if Greater Than or Equal To(a) & Sensitivity & 1 - Specificity \\
\hline 5.150 & 1.000 & 1.000 \\
\hline 6.225 & 1.000 & .972 \\
\hline 6.650 & 1.000 & .944 \\
\hline 7.050 & 1.000 & .917 \\
\hline 7.150 & 1.000 & .889 \\
\hline 7.225 & .981 & .861 \\
\hline 7.295 & .962 & .861 \\
\hline 7.370 & .943 & .861 \\
\hline 7.435 & .925 & .806 \\
\hline 7.475 & .925 & .778 \\
\hline 7.485 & .925 & .750 \\
\hline 7.545 & .925 & .722 \\
\hline 7.640 & .925 & .694 \\
\hline 7.690 & .906 & .694 \\
\hline 7.750 & .906 & 639 \\
\hline 7.850 & .906 & .611 \\
\hline 7.930 & .868 & .611 \\
\hline 8.010 & .868 & .583 \\
\hline 8.080 & .868 & .556 \\
\hline 8.150 & .868 & .528 \\
\hline 8.300 & .830 & .500 \\
\hline 8.435 & .811 & .500 \\
\hline 8.480 & .811 & .472 \\
\hline 8.545 & .811 & .444 \\
\hline 8.700 & .811 & .333 \\
\hline 8.805 & .792 & .306 \\
\hline 8.855 & .755 & .306 \\
\hline 8.950 & .736 & .306 \\
\hline 9.050 & .698 & .306 \\
\hline 9.105 & .698 & .278 \\
\hline
\end{tabular}




\begin{tabular}{|c|c|c|}
\hline Positive if Greater Than or Equal To(a) & Sensitivity & 1 - Specificity \\
\hline 9.115 & 679 & .278 \\
\hline 9.155 & 679 & .250 \\
\hline 9.195 & .642 & .222 \\
\hline 9.205 & .623 & .222 \\
\hline 9.255 & .604 & .222 \\
\hline 9.345 & .604 & .194 \\
\hline 9.395 & .585 & .194 \\
\hline 9.415 & .547 & .167 \\
\hline 9.465 & .528 & .167 \\
\hline 9.550 & .491 & .167 \\
\hline 9.635 & .434 & .167 \\
\hline 9.685 & .396 & .167 \\
\hline 9.715 & .340 & .167 \\
\hline 9.745 & .340 & .139 \\
\hline 9.780 & .321 & .139 \\
\hline 9.825 & .302 & .139 \\
\hline 9.875 & .283 & .139 \\
\hline 10.000 & .245 & .139 \\
\hline 10.150 & .245 & .111 \\
\hline 10.250 & .226 & .056 \\
\hline 10.390 & .189 & .056 \\
\hline 10.485 & .170 & .056 \\
\hline 10.595 & .151 & .056 \\
\hline 10.820 & .132 & .056 \\
\hline 11.020 & .113 & .056 \\
\hline 11.250 & .094 & .056 \\
\hline 11.500 & .075 & .056 \\
\hline 11.750 & .075 & .028 \\
\hline 11.950 & .057 & .028 \\
\hline 12.450 & .057 & .000 \\
\hline 13.010 & .038 & .000 \\
\hline 13.160 & .019 & .000 \\
\hline 14.200 & .000 & .000 \\
\hline
\end{tabular}

Table 2:

\begin{tabular}{|c|c|c|c|c|c|}
\hline Studies & $\begin{array}{c}\text { Total } \\
\text { number }\end{array}$ & $\begin{array}{c}\text { Hyperdestructive } \\
\text { group }\end{array}$ & $\begin{array}{c}\text { Hypoproductive } \\
\text { group }\end{array}$ & Cut-off value for MPV \\
\hline Negash et al[ ${ }^{[5]}$ & 83 & 33 & 50 & $\begin{array}{c}<10.75 \text { for hypoproductive, } \\
>11.05 \text { for hyperdestructive }\end{array}$ & $<0.0001$ \\
\hline Farweez et al ${ }^{[6]}$ & 80 & 40 & 40 & $>9.7 f$ for hyperdestructive & $<0.001$ \\
\hline Gulati et al ${ }^{[7]}$ & 100 & 79 & 21 & $<10.75$ for hypoproductive, & $<0.005$ \\
\hline Parveen et al ${ }^{[8]}$ & 120 & 26 & 94 & $>11.05$ for hyperdestructive & $>0.05$ for hyperdestructive \\
\hline Our study & 89 & 53 & 36 & $>8.5$ for hyperdestructive & $<0.001$ \\
\hline
\end{tabular}




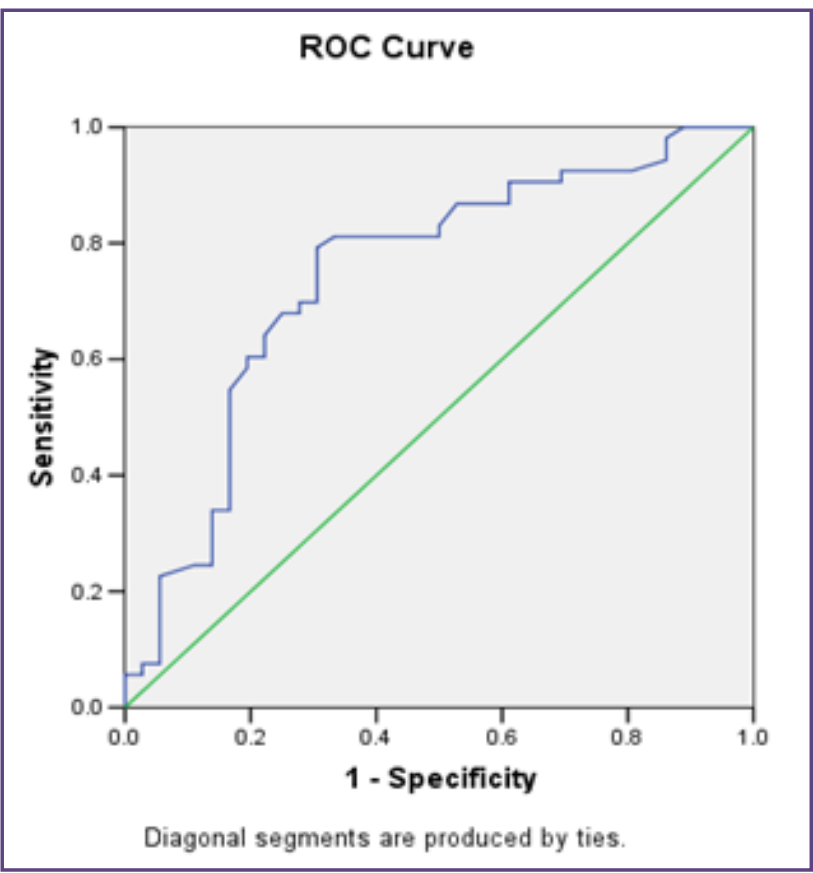

\section{Discussion}

Thrombocytopenia is a common clinical manifestation associated with various causes like infections, leukaemias, drugs, splenic sequestration, genetic conditions, autoimmune dieases, bone marrow infiltration, malignancies, decreased production, etc. Due to the automated analysers, the number of cases being diagnosed with thrombocytopenia is on an increasing trend.

With the newer automated analysers, parameters like the MPV, platelet large cell ration are

generated. Various studies have been done on MPV and thrombocytopenia, to find out the significance of MPV and to derive a cut-off value to distinguish between thrombocytopenia due to hyperdestructive and hypoproductive etiologies. These studies are summarised in the table (table 2) below. Note that all these studies have found significant association and cut-off value for MPV.

The etiologies and MPV have also been studied. In a study by Van der Lelie et al ${ }^{[9]}$ on patients with septicaemia, they found 13 of 25 cases with high MPV. They further added that the high MPV was not related to any particular microorganism.

The association between Dengue and MPV is variable as shown by studies. Studies by Reddy et al ${ }^{[10]}$ and Khanna et $\mathrm{al}^{\left[{ }^{[1]}\right]}$ have shown a raised MPV in dengue cases. However a study by Nehara et al ${ }^{[12]}$ has shown a low MPV in dengue cases.
MPV association with ischaemic heart disease has also been studied by some authors. A high MPV has been observed. ${ }^{[13,14]}$

A higher MPV was observed in patients with chronic urticaria, a dermatological disorder by Confino cohen et al. ${ }^{[15]}$

The utility of MPV in idiopathic thrombocytopenic purpura(ITP) is studied by many authors.

In a study by Ntaios et al ${ }^{[16]}$, they concluded that MPV at a cut-off of 9-10fl can reliably differentiate between ITP which is a hydestructive state from postchemotherapy myelosuppression which is a hypoproductive state.

Rajantie, Javela, Joutsi-Korhonen and Kekomaki studied various parameters in 21 children with chronic thrombocytopenia and found that increased megakaryocytosis at diagnosis was associated with larger MPV and higher percentage of reticulated platelets and not with platelet level or plasma thrombopoietin. ${ }^{[17]}$

In a study by Niethammer $\mathrm{A} \mathrm{G}$ and Forman $\mathrm{E} \mathrm{N}$ involving 156 children, they compared the MPV and the highest peak of the platelet volume distribution curve to differentiate between thrombocytopenia due to decreased production and increased destruction, they concluded that maximum of platelet histogram is better. ${ }^{[18]}$

Platelet indices are of help in conditions associated with thrombocytosis as well. In their article, Leader, Pereg and Lishner have stated that a normal platelet distribution width in thrombocytosis is highly suggestive of a reactive etilogy. ${ }^{[19]}$

The differences observed in the MPV cut-off values may be due to the different analysers used. Thrombocytopenia when coexisting with other diseases like endocrine dysfunction, autoimmune disorders, dermatologic disorders, ischaemic heart diseases, sepsis, etc may alter the MPV values.

\section{Conclusion}

We conclude that a MPV value of $>8.5 \mathrm{fl}$ is suggestive of a hyperdestructive etiology. Even though bone marrow remains a gold standard to distinguish between the hyperdestructive and hypoproductive thrombocytopenia groups, platelet indices like MPV can be used to aid in the diagnosis of etiology of thrombocytopenia.

\section{References}

1. Singh T, Singh N. Formation of blood cells: Bone marrow biopsy. In: Saxena R, Pati HP, Mahapatra M, editors. De Gruchy's Clinical Haematology in Medical Practice. 6th Adapted Edition, Ch. 1. New Delhi: Wiley; 2013. 
2. Giles C. The platelet count and mean platelet volume. Br J Haematol 1981;48:31-7.

3. Demirin H, Ozhan H, Ucgun T, Celer A, Bulur S, Cil H, Gunes $\mathrm{C}$, Yildirim HA. Normal range of mean platelet volume in healthy subjects: Insight from a large epidemiologic study. Thromb Res. 2011 Oct;128(4):358-60.

4. Numbenjapon T, Mahapo N, Pornvipavee R, Sriswasdi C, Mongkonsritragoon W, Leelasiri A, et al. A prospective evaluation of normal mean platelet volume in discriminating hyperdestructive thrombocytopenia from hypoproductive thrombocytopenia. Int J Lab Hematol 2008;30:408-14.

5. Negash M, Tsegaye A, Medhin AG. Diagnostic predictive value of platelet indices for discriminating hypo productive versus immune thrombocytopenia purpura in patients attending a tertiary care teaching hospital in Addis Ababa, Ethiopia. BMC Hematol. 2016;16(1):18.

6. Elsewefy D, Farweez B, Ibrahim R,. Platelet indices: consideration in thrombocytopenia. Egypt $\mathrm{J}$ Haematol . 2014;39(3):134.

7. Gulati I, Kumar H, Sheth J, Dey I. Diagnostic implication of mean platelet volume in thrombocytopenia. Med J DY Patil Univ 2017;10:370-5.

8. Parveen S, Vimal M. Role of Platelet Indices in Differentiating Hypoproductive and Hyperdestructive Thrombocytopenia. Annals of Pathology and Laboratory Medicine, 2017; 4(3) :4-7.

9. Van der Lelie J, Von dem Borne AK. Increased mean platelet volume in septicaemia. J Clin Pathol 1983;36:693-6.

10. Reddy RS, Phansalkar MD, Ramalakshmi PV. Mean platelet volume (MPV) in thrombocytopenia. J Contemp Med Dent 2014;2:45-50.
11. Khanna R, Nayak DM, Manohar C, Dhar M. A retrospective evaluation of mean platelet volume as a discriminating factor in thrombocytopenia of hypoproductive and hyperdestructive aetiologies. J Evol Med Dent Sci 2013;2:9059-65.

12. Nehara HR, Meena SL, Parmar S, Gupta BK. Evaluation of platelet indices in patients with dengue infections. Int J Sci Res 2016;5:78-81.

13. Shah A, Chaudhari SN, Shah MH. Role of platelet parameters in diagnosing various clinical conditions. Natl J Med Res 2013;3:162-5.

14. Ranjith MP, Divya R, Mehta VK, Krishnan MG, KamalRaj R, Kavishwar A. Significance of platelet volume indices and platelet count in ischaemic heart disease. J Clin Pathol 2009;62:830-3.

15. Confino-Cohen R, Chodick G, Shalev V, Leshno M, Kimhi O, Goldberg A. Chronic urticaria and autoimmunity: associations found in a large population study. J Allergy Clin Immunol (2012) 129(5):1307-13.

16. Ntaios G, Papadopoulos A, Chatzinikolaou A, Saouli Z, Karalazou P, Kaiafa G, et al. Increased values of mean platelet volume and platelet size deviation width may provide a safe positive diagnosis of idiopathic thrombocytopenic purpura. Acta Haematol 2008;119:173-7.

17. Rajantie J, Javela K, Joutsi K L, Kekomäki R. Chronic thrombocytopenia of childhood: Use of non-invasive methods in clinical evaluation. Eur J Haematol 2004;72:268-72.

18. Niethammer AG, Forman EN. Use of the platelet histogram maximum in evaluating thrombocytopenia. Am J Hematol 1999;60:19-23.

19. Leader A, Pereg D, Lishner M. Are platelet volume indices of clinical use? A multidisciplinary review. Ann Med 2012; 44:805-816.

*Corresponding author:

Dr. Archana Bhat, Assistant Professor, Dept of Pathology, Father Muller Medical College, Mangalore, Karnataka, India,

Phone: +91 9035572134

Email: archibhat3@gmail.com

Financial or other Competing Interests: None. 